\title{
Transmission System Planning Considering Solar Distributed Generation Penetration
}

\author{
Phillipe Vilaça Gomes ${ }^{1,2}$, João Tomé Saraiva ${ }^{1,2}$ \\ ${ }^{1}$ Faculdade de Engenharia da Universidade do Porto, Porto, Portugal. \\ 2 INESC TEC, Porto, Portugal. \\ email: phillipe.gomes@fe.up.pt, jsaraiva@,fe.up.pt
}

\begin{abstract}
In recent years, power systems have been watching important advancements related with Plug-in-Electrical Vehicles (PEVs), Demand Side Management (DSM), Distributed Generation (DG), Microgrid and Smart Grid installations that directly affect distribution networks while impacting indirectly on Transmission studies. These changes will lead to an extra flexibility on the transmission-distribution boundary and to a significant modification of the load patterns, that are an essential input to planning studies. In this scope, this paper describes a multiyear Transmission Expansion Planning (TEP) solved by Evolutionary Particle Swarm Optimization (EPSO) and incorporating the impact of solar DG penetration. The primary substation load profiles and the solar generation profiles are taken into account on the planning problem. The numerical simulations were conducted using the IEEE 24 bus reliability test system in which the planning horizon is 3 years and the load growth is $2.5 \%$ per year. If demand and solar DG peaks are coincident, then the liquid demand seen by the transmission network gets reduced enabling a reduction of investment costs. In the tested cases, these peaks were not coincident so that the optimal expansion plan remains unchanged even though the injected power from DG is large. This stresses the fact that solar DG may not on an isolated way contribute to alleviate the demand seen by transmission networks but should be associated with storage devices or demand side management programs.
\end{abstract}

Index Terms - Multiyear Transmission Expansion Planning, AC - Optimal Power Flow, Solar DG, Evolutionary PSO.

\section{INTRODUCTION}

The purpose of a TEP problem is to determine how a transmission system should evolve over time in the most economical way according to the planner's main drivers which, in the case, can correspond to the load growth, the connection of new generation sources or new demand centers, the equipment aging, the improvement of competition between generation companies, changes in export/import patterns between neighbor systems and variations in the supply reliability requirements of customers [1]. Therefore, using a pre-defined list of candidate equipments (transmission lines, cables, transformers, etc.) that can be inserted on the grid, the TEP problem aims at identifying the ones to be built and their commissioning date to attend a pre fixed objective (investment cost, operational cost, GHG emissions, reliability, etc.).

The TEP problem has non-linear and non-convex nature which leads to a huge computational effort. In order to overcome this burden, relaxed models are often used as static approaches or formulations based on the DC power flow. Although their solutions are considered reasonable in the literature, these models don't incorporate a holistic view over the entire planning problem, the reactive power, the branch losses and the voltage limits on the bars are often disregarded, and so its computational effort is lighter. It is then clear that these models don't represent the real world problem so that less realistic solutions can be obtained. In alternative, the original problem should be considered eventually using bioinspired metaheuristics to solve it (instead of traditional mathematical optimization techniques). This kind of tools are able to give optimal or suboptimal solutions taking advantage of patterns recognized in the nature as behaviors of fireflies, bats, ants and swarms for instance.

In order to solve the TEP problem, and apart from other considerations regarding the evolution over time of other input variables (generation, equipments, etc.), we usually consider a scenario for the system demand for each year of the planning horizon. For each of these years and for the transmission assets available in that year an optimal power flow analysis should be done in order to check if the system operates properly, i.e., if there is no congestion on the lines, if Power Not Supplied (PNS) is zero and if the voltage profile is adequate. These demand scenarios can then be addressed using probabilistic or deterministic approaches or considering uncertainties representing vague information provided by the planner for instance under the form of fuzzy concepts. Regarding deterministic approaches, it is typically considered the forecasted annual peak demand as the demand scenario that the system must supply in a secure and reliable way.

The advent of changes namely in distribution networks related with the increasing presence of DG, the development of micro and smart grids and the increasing number of PEV's must also be incorporated in long-term expansion planning methodologies in view of their impact on the network depending on regional incentive policies. In this paper, we 
considered a deterministic load scenario taking into account an increased penetration of solar DG on the IEEE 24 bus RTS in order to investigate how the deployment of this type of DG impacts the definition of the transmission expansion plans. The investigation of this impact can therefore be considered as the main contribution of this paper.

Regarding the structure of the paper, following this Introduction, Section II reviews some concepts related with distributed generation, Sections III presents the mathematical formulation of the dynamic TEP problem and Section IV details the main blocks of the EPSO algorithm. Finally, Section $\mathrm{V}$ addresses the methodology used in this paper. Section VI presents the simulation results and Section VII draws the main conclusions of this research.

\section{DISTRIBUTED GENERATION}

Distributed generation is usually as associated to small generation units connected to the power grid either on the customer side or to the distribution network [2]. The size of a typical DG unit ranges from $1 \mathrm{~kW}$ to 10 or $20 \mathrm{MW}$ but in some cases, as for large wind parks or solar PV stations, it can reach 100 or $200 \mathrm{MW}$. Besides, regarding to its primary source, DG can be classified as renewable (solar, wind, hydro) or nonrenewable (internal combustion engine) energy stations.

The exploitation of energy generation from renewable sources has been increasing in several countries, in most cases induced by regulatory incentives for small distributed generation such as Feed-in Tariffs and Net Metering schemes.
These incentives are justified by the potential benefits that the deployment of these distributed sources can bring to entire system and to the society as a whole. According to [3] these advantages are related with the possible postponement of expansion investments in distribution and transmission systems, the reduction of the environmental impact, the reduction of the network loading levels, the reduction of losses and the diversification of energy sources.

Although the solar DG penetration is still low in most countries, the global incident solar radiation can reach large values ranging from 900 to $1250 \mathrm{kWh} / \mathrm{m}^{2}$ throughout the year in Germany, $900-1650 \mathrm{kWh} / \mathrm{m}^{2}$ in France, $1200-1850 \mathrm{kWh} / \mathrm{m}^{2}$ in Spain and 4200-6700 kWh/m $\mathrm{m}^{2}$ in Brazil. Therefore, several studies investigate the barriers to DG penetration [4] as well as its impact on the grids [5].

In TEP problems DG is usually modeled as a negative real load, that is, the DG reduces locally the real load on a primary substation. Fig. 1 shows this behavior for a bus over one day (24 hours) on the test system used in this paper. The new demand that will be used to carry out the expansion planning studies takes into account the solar DG penetration. In this example, solar generation starts at 6 am and ends at $6 \mathrm{pm}$ and has a generation peak at 12:00. It should be stressed that the expansion study should continue to be done in a way to ensure the safe operation during the peak demand (19:00), and this drive is not changed while considering the injection of electricity from PV sources on the grid nodes.

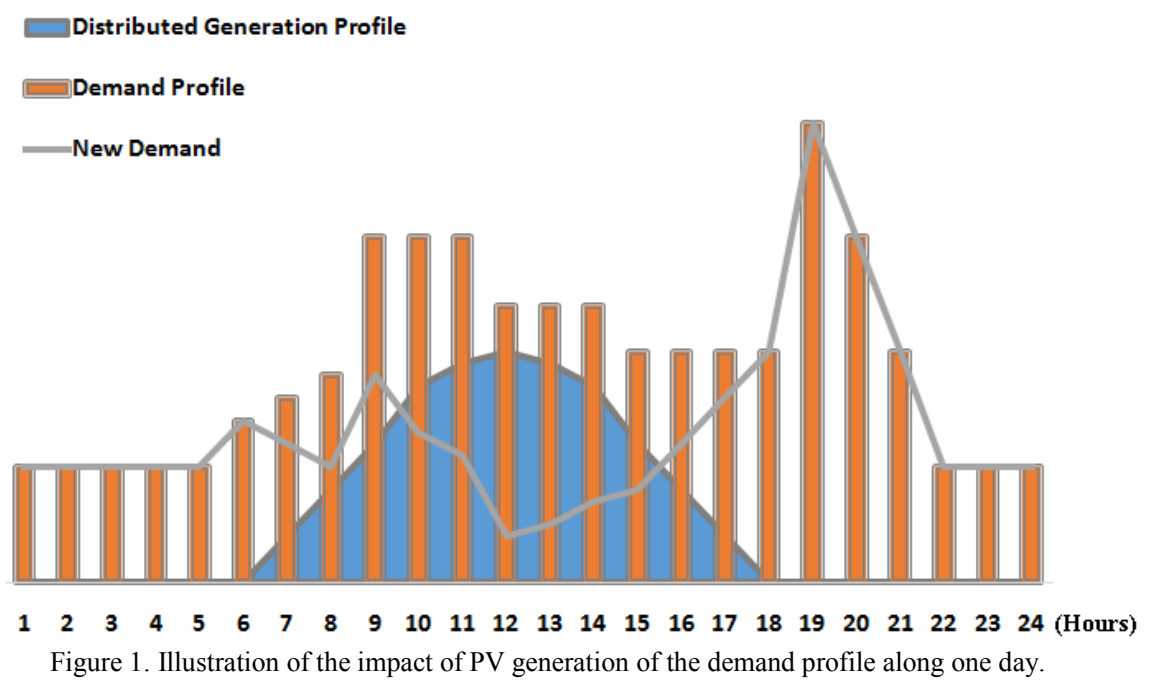

\section{TEP MATHEMATICAL FORMULATION}

The mathematical formulation adopted in this paper for the TEP problem takes into account the investment cost $\left(C_{i n v, p}\right)$ and a penalization term ( $\beta$ ) for Power Not Supplied (PNS). This means that one possible expansion plan is characterized by the investment cost associated to the insertion of new equipments on the grid on specific years and by the expected PNS in each year of the horizon when the annual peak load is applied on the system. A solution is considered feasible when it is able to ensure a safe operation of the system over the planning horizon, that is, it displays zero PNS in every year of the horizon. Furthermore, the formulation includes physical constraints related with the branches and generator capacity limits, financial limitations related with the capital available in each year or along the entire planning horizon and quality of supply constraints associated to reliability indices. According to these ideas, the associated TEP problem can be formulated by (1) to (4).

$$
\text { Minimize } \sum_{p=1}^{n p} \kappa_{p} \cdot C_{i n v, p}+\beta \cdot P N S
$$

Subject to: 


\section{Physical Constraints \\ Financial Constraints \\ Quality of service Constraints}

In this formulation $\kappa_{p}$ is the present-worth value coefficient given by (5), $\mathrm{d}$ is the discount rate and $\mathrm{p}$ is the index associated to each period in the planning horizon.

$$
\kappa_{p}=\frac{1}{(1+d)^{p}}
$$

Although the AC power flow (AC-OPF) requires a larger computational burden, this is the most adequate model to deal with TEP problems because it considers the reactive power, the losses and the bus voltage limits. Therefore, we used an AC-OPF based model in order to compute the PNS when the system supplies the annual peak demand for each planning year and incorporating the new equipments included in the expansion plan. Given the fact that the AC-OPF problem has to be solved a large number of times, associated to the years in the planning horizon and with the trial expansion plans to test, we used the MATPOWER tool [6] to assist the solution of the mentioned AC-OPF problem.

The AC-OPF used in this paper is formulated by (6) to (14): For a given trial expansion plan being tested, this problem is solved for each year in the planning horizon considering in each year the new equipments that are included in that solution until the year under analysis.

\section{Min PNS}

subject to

$$
\begin{gathered}
P(V, \theta, n)-P_{G}+P_{D}=0 \\
Q(V, \theta, n)-Q_{G}+Q_{D}=0 \\
P_{G \min } \leq P_{G} \leq P_{G \max } \\
Q_{G \min } \leq Q_{G} \leq Q_{G \text { max }} \\
V_{\min } \leq V \leq V_{\max } \\
(N+\stackrel{o}{N}) S^{\text {from }} \leq(N+\stackrel{o}{N}) S_{\max } \\
(N+\stackrel{o}{N}) S^{t o} \leq(N+\stackrel{o}{N}) S_{\max } \\
0 \leq n \leq n_{\max }
\end{gathered}
$$

In this formulation, the objective function (6) corresponds to the minimization of PNS, $P_{G}$ and $Q_{G}$ are the active and reactive power generation, $P_{D}$ and $Q_{D}$ are the active and reactive power demand, $V$ is the voltage magnitude, $S_{i j}^{\text {from }}$ and $S_{i j}^{t o}$ are the apparent flows in branch ij, $N$ and $\stackrel{o}{N}$ are diagonal matrixes containing the inserted equipments and the base topology equipments.

The solution having the lowest value for Eq. (1) is considered the optimal solution and will be analyzed considering issues as the operation costs, reliability, losses and environmental impact.

\section{EPSO ALGORITHM}

EPSO is a powerful tool that combines concepts of evolutionary computation and multi agent population taking advantage of the standard blocks that are typical in Genetic Algorithm and in Particle Swarm Optimization. This tool is able to combine the best features of these two groups of techniques and therefore has excellent performance in solving complex problems such as the one addressed in this paper.

This algorithm is based on the evolution of a set of particles, each of them representing solutions for the problem. Along the evolution process the particles evolve according to a fitness function and continue to improve in each iteration until the process reaches a pre-established stopping criterium, and the best solution of the last population is provided to the user. Fig. 2 details the main blocks of the EPSO algorithm that will also be described in the next paragraphs.

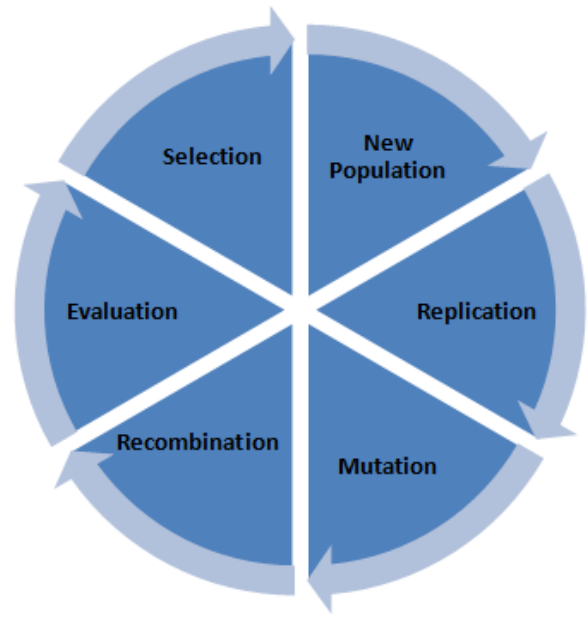

Figure 2. Main blocks of the EPSO Algorithm.

The initial population is created randomly and the developed approach uses a Tabu List to prevent repeating the same particle in the population, that is, to increase the diversity of the particles in the initial population. In the replication block each population is cloned $r$ times in order to create new populations that will be mutated in the next block. In the mutation block the weights and the best particle found until now (gbest) for all the populations are mutated using (15) and (16) in which the symbol * denotes the mutation operator. This process increases the diversity of the individuals under analysis.

$$
\begin{aligned}
& w_{i j}^{*}=0,5+\operatorname{rand}()-\frac{1}{1+\exp \left(-w_{i j}^{i t}\right)} \\
& \text { gbest }=\text { gbest }+\operatorname{round}\left(2 \cdot w_{i 4}^{*}-1\right)
\end{aligned}
$$

Therefore, new populations (offsprings) are created in the recombination block based on the PSO movement rule. According to (17) the position of a particle $\mathrm{i}$ in iteration it +1 is the result of its position in iteration it plus the velocity vector 
given by (18). This procedure is repeated for all particles in the cloned populations.

$$
\begin{gathered}
x_{i}^{i t+1}=x_{i}^{i t}+v_{i}^{i t+1} \\
v_{i}^{i t+1}=w_{i 1}^{*} \cdot v_{i}^{i t+1}+w_{i 2}^{i t+1} \cdot\left(\text { pbest }_{i}-x_{i}^{i t}\right)+w_{i 3}^{i t+1} \cdot\left(\text { gbest }-x_{i}^{i t}\right) \cdot P
\end{gathered}
$$

The first term in (18) represents the inertia of the particle, the second term represents its individual knowledge and the last term represents the collective knowledge of the swarm. $\mathrm{P}$ is the communication factor described in [7]. It typically takes values 0 or 1 so that if 0 is used for a position of the particle vector then the collective knowledge is not passed to this particle in the next iteration. The evaluation block checks if a candidate plan ensures a safe operation (without PNS) and so its load shedding cost is zero. Otherwise the PNS is multiplied by a penalty factor (in $\$ / M W$ ). Once this step is finished, all the particles are characterized by their investment and expected load shedding costs. In the selection block a tournament selection is performed to build the new population having the same size of the initial one. The iterative process continues until the best solution remains unchanged along a pre-defined number of iterations. In the selection block it was also used a Tabu List in order to ensure the diversity of the new population. This list checks if one solution is already in the population. If so it modifies this solution selecting randomly one investment in this solution and postponing it by moving it one year ahead.

TEP problems have integer nature which leads to the phenomenon of combinatorial explosion of investment alternative plans. This characteristic typically leads to a high computational effort to identify good quality plans. In order to tackle this problem, the described EPSO algorithm was implemented using parallel computing.

\section{Methodology}

In this paper the penetration of solar DG was organized in 4 scenarios: $0 \%, 10 \%, 15 \%$ and $20 \%$ of the annual peak demand connected to each bus. After the optimization process carried out by the EPSO algorithm is done (considering the new peak demand for each scenario), the DG impact is analyzed considering four items: reliability, economic aspects, losses and environmental aspects. The probabilistic index Expected Energy Not Supplied (EENS) is used to measure the system reliability. EENS reflects not only the base topology and physical limits of the system under analysis but it also incorporates uncertainties associated with the non-ideal behavior of system components. In order to estimate the EENS it is not enough to sample components out of service. In fact it is necessary to sample operation and repair times of the components in order to access the duration of each state which justifies using a chronological Monte Carlo as detailed in [8].

Regarding the economic analysis, four load blocks representing one day of each season of the year are used in order to estimate the operation costs. Each primary substation (represented by the buses of the system) have their own load profile and these values can be assessed in [9] taking into account the Monday of the 2nd week for the Winter, the Saturday of the 13th week for the Spring, the Friday of the 24th week for the Summer and the Sunday of the 41st week for Autumn. The methodology discussed in this paper is illustrated in Fig. 3.

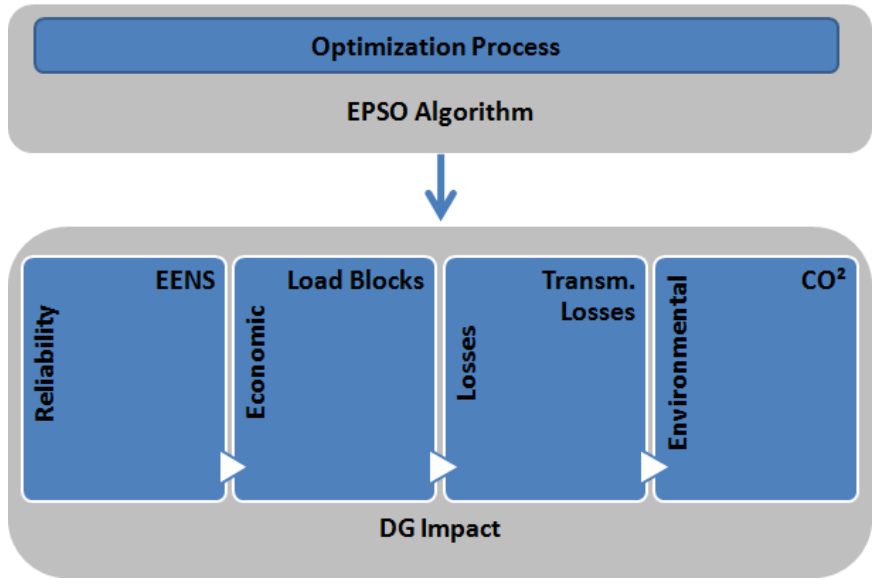

Figure 3. Methodology for DG impact on TEP

As an example, Fig. 4 represents the new load profile for each season of the primary substation connected to bus 1 assuming a $10 \%$ DG penetration (as suggested by Fig. 1). In this paper, the maximum solar generation (solar peak at 12:00) for each bar is assumed to be proportional to its annual peak load. As an example, Fig. 5 presents the DG generation in MW in each bus for the three penetration levels that were considered, $10 \%, 15 \%$ and $20 \%$. As a whole, the $10 \%$ level of penetration is associated to an installed capacity of $570 \mathrm{MW}$, $15 \%$ is associated to $855 \mathrm{MW}$ and $20 \%$ to $1140 \mathrm{MW}$ for the annual peak demand of $5700 \mathrm{MW}$ considered in the first year.

\section{RESULTS}

This section presents the results obtained using the proposed TEP approach when applied to a modified version of the IEEE RTS 24 bus system [9]. The system that was used has some differences regarding the original one, as described below:

i. the loads are modeled as negative real power injections with associated negative costs as described in [10];

ii. the values of all the loads were duplicated and the installed capacity of all generators were tripled (real and reactive) in order to turn the transmission network more stressed;

iii. the modified system has 9 hydro generators that have initial maximum capacity of $2880 \mathrm{MW}(28,2 \%$ of the total capacity of the system) as follows:

- 6 hydro generators of $150 \mathrm{MW}$ in bus 22;

- 1 hydro generator of $1050 \mathrm{MW}$ in bus 23;

- 1 hydro generator of $465 \mathrm{MW}$ in bus 15 ;

- 1 hydro generator of $465 \mathrm{MW}$ in bus 16 ; 


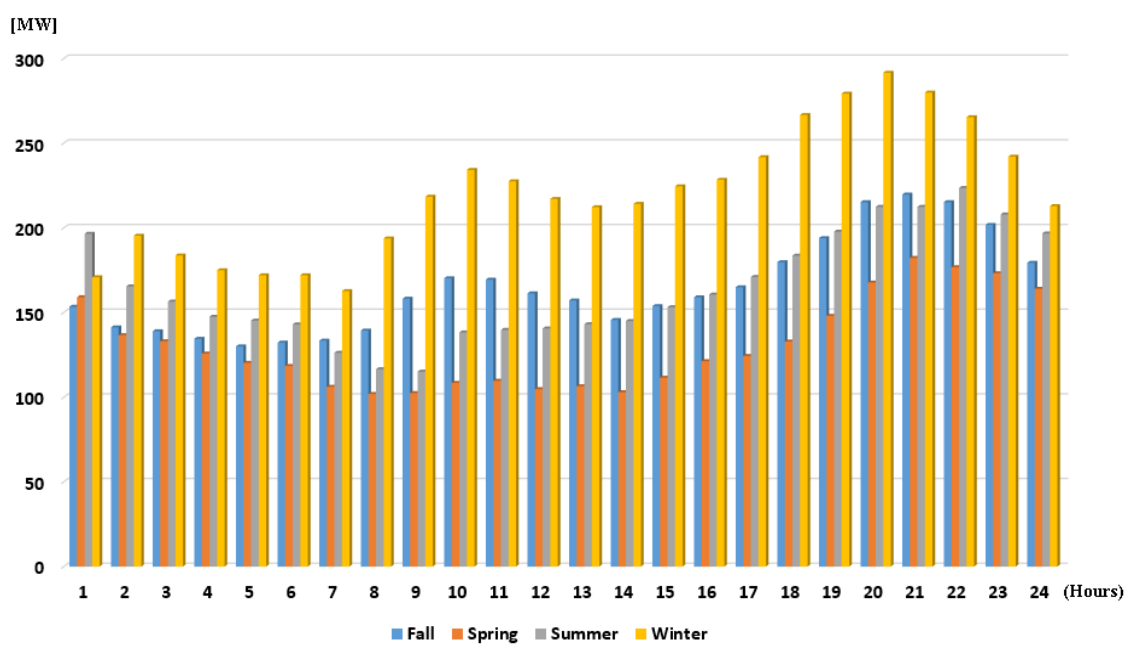

Figure 4. New load profile for the bus 1 (with 10\% DG penetration).

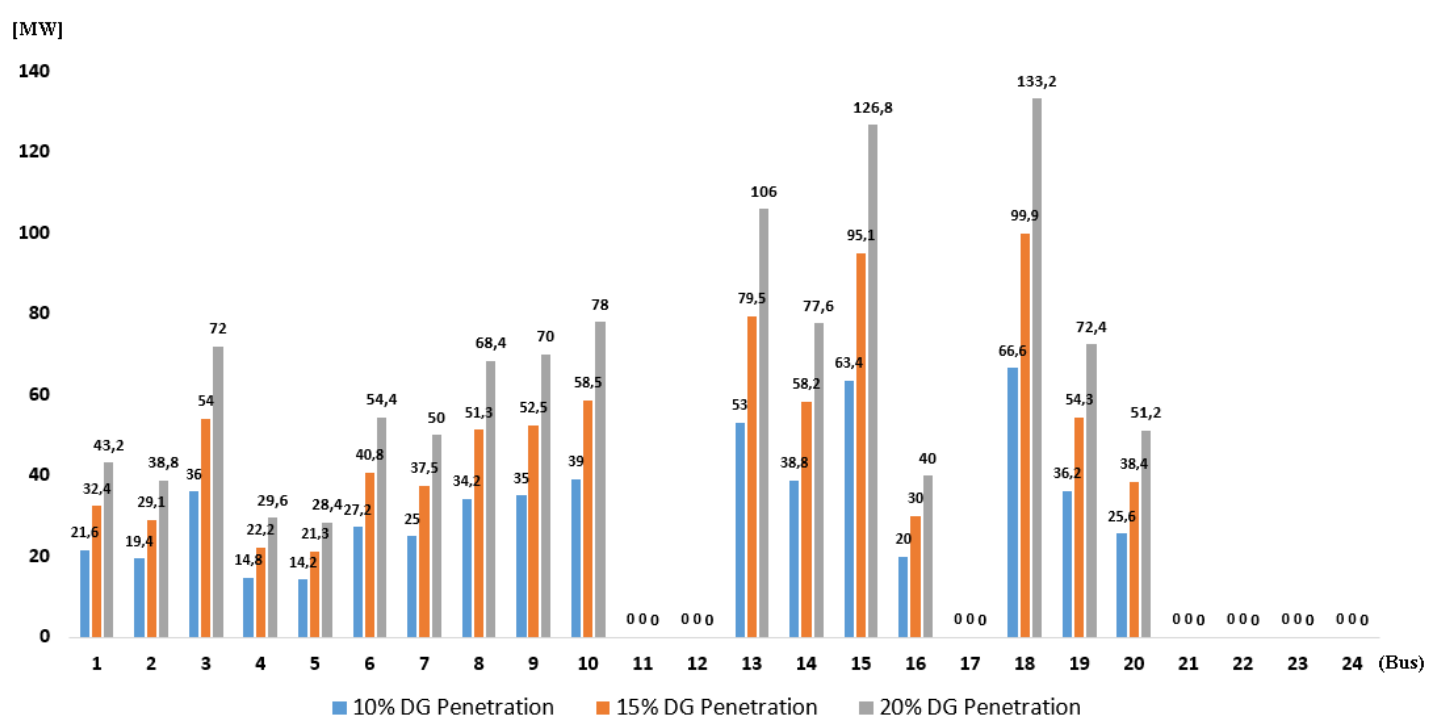

Figure 5. DG penetration by bus and scenario at $12 \mathrm{am}$ (Peak of solar generation).

The initial peak demand is $5700 \mathrm{MW}$ and the total generation capacity is $10215 \mathrm{MW}$. Regarding the $\mathrm{CO}_{2}$ emissions, we use data from [11]. The load growth was set at $2,5 \%$ per year and the discount rate was set at $5 \%$ per year, the number of particles in each population of the EPSO algorithm was set at 50, the planning horizon is 3 years and the PNS penalization cost was set at $10^{9} \$ / \mathrm{MW}$. The EPSO algorithm stops after running 50 iterations with the same best solution. The simulations were run in MATLAB with an Intel i7, $3.4 \mathrm{GHz}, 8 \mathrm{~GB}$ RAM.

As the annual peak load for this system occurs after $6 \mathrm{pm}$, the solar distributed generation has no impact in decreasing it since no associated storage devices are being considered. Additionally, the optimization process takes into account only the minimization of the investment cost for new equipments on the grid while ensuring that the annual peak demand is supplied. Therefore, for the considered cases (solar DG penetration of $0 \%, 10 \%, 15 \%$ and $20 \%$ ) the annual peak demand is the same which means that the optimal expansion plan identified by the EPSO algorithm remains unchanged. This plan was obtained running 112 iterations in 42 minutes. The evolution of the solution is shown in Fig. 6 below and for each analyzed case Table I provides the values of the indices used to analyze the obtained expansion plan considering the DG penetrations that were tested.

The best solution found by EPSO algorithm corresponds to the installation of:

- Year 1 - a transformer between bus 3 and 24, one $138 \mathrm{kV}$ cable connecting bus 6 to 10 , one $138 \mathrm{kV}$ line connecting buses 7 to 8 ;

- Year 2 - one $138 \mathrm{kV}$ line connecting buses 1 to 5 in the second year.

These equipments allow the system to gain flexibility 
enough to accommodate the demand in Period 3 so that no new equipment is required in this period.

Table 1: Comparison of the results for the 4 DG penetration scenarios.

\begin{tabular}{|c|c|c|c|c|c|c|}
\hline Cases & $\begin{array}{c}\text { DG } \\
\text { penetration }\end{array}$ & $\begin{array}{l}\text { Inv. Cost } \\
\left(10^{6} \text { US\$) }\right.\end{array}$ & $\begin{array}{c}\text { Op. } \text { Cost } \\
\left(10^{6} \text { US\$ }\right)\end{array}$ & $\begin{array}{c}\text { EENS } \\
\text { (MWh/year) }\end{array}$ & $\begin{array}{c}\text { Trans. Losses } \\
\text { (MW) }\end{array}$ & $\begin{array}{c}\mathrm{CO}_{2} \text { Emissions } \\
\left(\mathrm{t} \mathrm{CO}_{2}\right)\end{array}$ \\
\hline 1 & $0 \%$ & 98.05 & 47.60 & 38295,98 & 14808.07 & 62131.47 \\
\hline 2 & $10 \%$ & 98.05 & 39.08 & 29563,18 & 14072.61 & 51495.52 \\
\hline 3 & $15 \%$ & 98.05 & 35.43 & 27126,98 & 13690.19 & 47620.69 \\
\hline 4 & $20 \%$ & 98.05 & 32.47 & 25739,72 & 13372.85 & 43649.91 \\
\hline
\end{tabular}

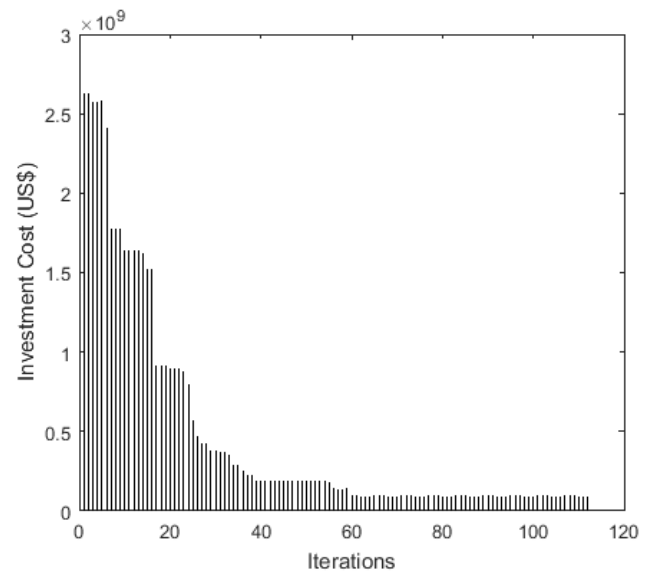

Figure 6. Evolution of the best particle of the EPSO Algorithm.

\section{CONCLUSIONS}

This paper describes a multiyear transmission expansion planning considering solar distributed generation penetration. The planning problem is conducted considering the investment cost and a penalization for Power Not Supplied over the planning horizon. On the other hand, Evolutionary Particle Swarm Optimization is used to solve the optimization problem via a parallel computing approach as a way to reduce the computation time. The develop approach models solar distributed generation as negative loads in each bar considering the solar penetration proportional to the demand.

The main motivation to develop this TEP approach comes from the need to consider in the transmission planning task the new load behavior present in the transmission-distribution boundary. The new load profiles are becoming increasingly affected by Plug-in-Electrical Vehicles, Demand Side Management Programs, Distributed Generation (DG) penetration and Microgrid and Smart Grid installations. Although affecting in a direct way distribution networks, these aspects impact indirectly on the transmission grids.

The results presented in Table I indicate that the penetration of solar distributed power generation can provide a better and safer operation for the system while reducing the operation costs, the transmission losses and the $\mathrm{CO}_{2}$ emission level while contributing to diversify the generation mix. In this case the peak of injected solar DG is not coincident with the annual demand peak period which means that the annual peak demand seen by the transmission network remains unchanged. As a result, even if the injected solar DG is increased to $20 \%$ of the peak demand in each bus, the optimal expansion plan remains unchanged. This suggests that solar DG is not able in an isolated way to reduce the liquid demand seen by transmission networks and thus contribute to postpone transmission investments and reduce the corresponding cost. Therefore, as main conclusion of this paper, solar DG should be associated to storage devices or demand side management programs should be implemented in order to reduce the investment effort in transmission networks.

\section{ACKNOWLEDGMENT}

The first author thanks CAPES Foundation, Ministry of Education of Brazil, for financing this research.

\section{REFERENCES}

[1] W. Li, Probabilistic transmission system planning. Wiley-IEEE Press, 2011.

[2] J. H. Zhao, J. Foster, Z. Y. Dong, and K. P. Wong, "Flexible Transmission Network Planning Considering Distributed Generation Impacts," IEEE Trans. Power Syst., vol. 26, no. 3, pp. 1434-1443, Aug. 2011.

[3] R. S. Maciel, M. Rosa, V. Miranda, and A. Padilha-Feltrin, "Multiobjective evolutionary particle swarm optimization in the assessment of the impact of distributed generation," Electr. Power Syst. Res., vol. 89, pp. 100-108, Aug. 2012.

[4] P. Dondi, D. Bayoumi, C. Haederli, D. Julian, and M. Suter, "Network integration of distributed power generation," J. Power Sources, vol. 106, no. 1, pp. 1-9, 2002.

[5] S. Haffner, L. F. A. Pereira, L. A. Pereira, and L. S. Barreto, "Multistage Model for Distribution Expansion Planning With Distributed Generation-Part I: Problem Formulation," IEEE Trans. Power Deliv., vol. 23, no. 2, pp. 915-923, Apr. 2008.

[6] R. D. Zimmerman, C. E. Murillo-Sanchez, and R. J. Thomas, "MATPOWER: Steady-State Operations, Planning, and Analysis Tools for Power Systems Research and Education," IEEE Trans. Power Syst., vol. 26, no. 1, pp. 12-19, Feb. 2011.

[7] V. Miranda, H. Keko, and Á. J. Duque, "Stochastic Star Communication Topology in Evolutionary Particle Swarms (EPSO)," Int. J. Comput. Intell. Res., vol. 4, no. 2, p. 105, 2008.

[8] P. Vilaça Gomes, J. Silva, and J. T. Saraiva, "Multiyear and MultiCriteria AC Transmission Expansion Planning Model Considering Reliability and Investment Costs," in IEEE EEM Porto 2016, 2016.

[9] P. Subcommittee, "IEEE Reliability Test System," IEEE Trans. Power Appar. Syst., vol. PAS-98, no. 6, pp. 2047-2054, Nov. 1979.

[10]P. V. Gomes and J. T. Saraiva, "Hybrid Discrete Evolutionary PSO for AC Dynamic Transmission Expansion Planning," in IEEE Energycon conference, 2016.

[11]C. A. Correa, R. Bolanos, A. Garces, and A. Molina, "Multiobjective environmental transmission network expansion planning," in 2013 IEEE Grenoble Conference, 2013, pp. 1-5. 